\title{
Liver cancer cells are sensitive to Lanatoside $C$ induced cell death independent of their PTEN status
}

\author{
Irem Durmaz ${ }^{\mathrm{a}}$, Ebru Bilget Guven ${ }^{\mathrm{a}}$, Tulin Ersahin ${ }^{\mathrm{b}}$, Mehmet Ozturk ${ }^{\mathrm{c}}$, Ihsan Calis ${ }^{\mathrm{d}}$, \\ Rengul Cetin-Atalay ${ }^{\mathrm{a}, \mathrm{b}, *}$
}

a Department of Molecular Biology and Genetics, Faculty of Science, Bilkent University, Ankara, Turkey

${ }^{\mathrm{b}}$ Cancer Systems Biology Laboratory, Graduate School of Informatics, METU, 06800 Ankara, Turkey

' Izmir Biomedicine and Genome Center, Faculty of Medicine, Dokuz Eylul University, Izmir, Turkey

${ }^{d}$ Department of Pharmacognosy, Faculty of Pharmacy, Near East University, Lefkosa (Nicosia), N. Cyprus

\section{A R T I C L E I N F O}

\section{Article history:}

Received 13 August 2015

Revised 6 November 2015

Accepted 14 November 2015

\section{Keywords:}

Lanatoside C

Oxidative stress

ERK

Akt

PTEN

Hepatocellular carcinoma

\begin{abstract}
A B S T R A C T
Background: Hepatocellular carcinoma is the second deadliest cancer with limited treatment options. Loss of PTEN causes the P13K/Akt pathway to be hyperactive which contributes to cell survival and resistance to therapeutics in various cancers, including the liver cancer. Hence molecules targeting this pathway present good therapeutic strategies for liver cancer.

Hypothesis: It was previously reported that Cardiac glycosides possessed antitumor activity by inducing apoptosis of multiple cancer cells through oxidative stress. However, whether Cardiac glycoside Lanatoside C can induce oxidative stress in liver cancer cells and induce cell death both in vitro and in vivo remains unknown. Methods: Cell viability was measured by SRB assay. Cell death analysis was investigated by propidium iodide staining with flow cytometry and PARP cleavage. DCFH-DA staining and cytometry were used for intracellular ROS measurement. Protein levels were analyzed by western blot analysis. Antitumor activity was investigated on mice xenografts in vivo.

Results: In this study, we found that Cardiac glycosides, particularly Lanatoside C from Digitalis ferruginea could significantly inhibit PTEN protein adequate Huh7 and PTEN deficient Mahlavu human liver cancer cell proliferation by the induction of apoptosis and G2/M arrest in the cells. Lanatoside $\mathrm{C}$ was further shown to induce oxidative stress and alter ERK and Akt pathways. Consequently, JNK1 activation resulted in extrinsic apoptotic pathway stimulation in both cells while JNK2 activation involved in the inhibition of cell survival only in PTEN deficient cells. Furthermore, nude mice xenografts followed by MRI showed that Lanatoside $\mathrm{C}$ caused a significant decrease in the tumor size. In this study apoptosis induction by Lanatoside $\mathrm{C}$ was characterized through ROS altered ERK and Akt pathways in both PTEN adequate epithelial and deficient mesenchymal liver cancer cells.

Conclusion: The results indicated that Lanatoside C could be contemplated in liver cancer therapeutics, particularly in PTEN deficient tumors. This is due to Lanatoside C's stress inducing action on ERK and Akt pathways through differential activation of JNK1 and JNK2 by GSK3 $\beta$.
\end{abstract}

(C) 2016 Elsevier GmbH. All rights reserved.
Abbreviations: HCC, hepatocellular carcinoma; DCFH-DA, dichlorofluorescin diacetate; DMEM, Dulbecco's modification of Eagle's medium; EMT, epithelial mesenchymal transition; JNK, c-Jun N-terminal kinase; MRI, magnetic resonance imaging; PARP, poly(ADP-ribose) polymerase; PI3K, phosphatidylinositol-4,5-bisphosphate 3-kinase; PTEN, phosphatase and tensin homologue; ROS, reactive oxygen species; RTK, receptor tyrosine kinase.

* Corresponding author at: Cancer Systems Biology Laboratory, Graduate School of Informatics, METU, 06800 Ankara, Turkey. Tel.: +90 312210 7887; fax: +90 312210 3745 .

E-mail addresses: durmaz@bilkent.edu.tr (I. Durmaz), bilget@bilkent.edu.tr (E.B. Guven), ersahin@metu.edu.tr (T. Ersahin), mehmet.ozturk@deu.edu.tr (M. Ozturk), icalis664@gmail.com (I. Calis), rengul@metu.edu.tr, rcatalay@gmail.com (R. CetinAtalay).

\section{Introduction}

Hepatocellular carcinoma is the sixth most common and one of the most fatal cancer type worldwide (Ferlay et al. 2012). Furthermore, incidence of HCC has been recently increased among individuals with obesity related liver disease in developed countries. Although the etiological factors that are thought to be fundamentally related to HCC are well-known (such as viral infections, toxic injury and recently obesity), there are still limited treatment options (Baffy et al. 2012). This is due to the fact that liver cancer cells are extremely resistant to conventional chemotherapy and radiotherapy. With the exception of Sorafenib, which generated notably modest clinical response, no well-known target-specific drug provided 
satisfactory clinical response to HCC in clinical assays (El-Serag et al. 2008). The five-year survival rate of HCC is only about seven percent and the recurrence rate is very high. Therefore, it is essential to recommend novel preventive or therapeutic approaches towards HCC (Yau et al. 2009).

Cancer cells acquire survival capacities by altering more than a single protein/pathway in parallel. Hence in these cancerous cells, there are proteins which exhibit altered functions in favor of cell survival and anti-proliferative cellular activities. Therefore, molecules targeting these signaling pathways are good candidates for targeted therapies (Ersahin et al. 2015). A good example to the alteration of mechanism in cancer cells would be the hyper-activation of the PI3K/Akt signaling pathway and hyperactivity of this pathway is frequently associated with numerous cancer types. PI3K/Akt pathway is involved in cell survival, proliferation, growth and metabolism and hence any changes in this pathway can alter key mechanisms required for cell survival and lead to various cancers (Ke et al. 2011). In HCC, constitutively active PI3K/Akt signaling due to loss of tumor suppressor protein PTEN is significantly associated with aggressive tumor behavior (Buontempo et al. 2011). Activation of PI3K/Akt/GSK$3 \beta$ pathway in several cases of liver cancer was reported to induce EMT which promoted tumor invasion and metastasis (Shearn et al. 2011). Ras/Raf/MEK/ERK pathway, which also controls of cell survival mechanisms in liver cancer, has cross-talks with PI3K/Akt/GSK-3 $\beta$ pathway. Activation of ERK protein has dual effects on cell survival and death. Stress induced ERK activation can also promote cell death; therefore in some conditions where there are cross-talks between these pathways, Akt and ERK proteins have anti- and pro-apoptotic actions respectively (Cagnol and Chambard 2010).

Cardiac glycosides are steroid-like compounds and their main source is the Digitalis genus and many cell-signaling mechanisms have been described for their anticancer activities (Prassas and Diamandis 2008). One of the main mechanisms is based on the inhibition of $\mathrm{Na}^{+} / \mathrm{K}^{+}$-ATPase by cardiac glycosides and involves Ras pathway activation, which leads to the release of ROS, resulting in apoptosis. Bufalin, a member of cardiac glycoside family, was previously shown to induce ROS and SAPK/JNK pathway activation in human colon cancer cells (Xie et al. 2011). Another cardiac glycoside ouabain was also described to induce ROS in lung cancer cells, activate JNK and PI3K/Akt signaling in myocyte proliferation (Liu et al. 2007). Even though there are various studies, which show the effects of these natural drugs in various cancer cells, their effects on HCC are still not known. In this study, cytotoxicities of pure glycosides, which were originated from $D$. ferruginea, in liver cancer cells were investigated. The study also analyzed the underlying molecular cellular pathways for this activity in drug resistant PTEN deficient mesenchymal like Mahlavu liver cancer cells and drug sensitive PTEN adequate epithelial like Huh7 cells (Buontempo et al. 2011). It was demonstrated that ROS induced actions of glycosides alternatively modified Akt and ERK pathways in these two cells with different genetic backgrounds.

This study investigates the antitumor activity of cardiac glycosides, particularly Lanatoside C, in liver cancer cells both in vitro and in vivo. For this purpose, initially the cytotoxic activities of Lanatoside $\mathrm{C}$ along with other glycosides were tested on liver cancer cells. Lanatoside $C$ was further analyzed at molecular level in order to identify the cellular pathways involved in this activity. Finally, the antitumor activity was exploited in nude mice xenograft models with PTEN deficient Mahlavu cell lines.

\section{Materials and methods}

\section{Extraction and fractionation of glycosides}

Glycosides were obtained from Digitalis ferruginea (also known as "rusty foxglove", Aladağ - Bolu, Turkey). Initial and majority of in vitro experiments were done with pure Lanatoside $C(D$. ferruginea) and further in vivo and in vitro studies were performed with Lanatoside C from Sigma Aldrich ( $\geq 95 \%$ purity by TLC, CID 656630 ) for versatility. Extraction and fractionation of cardioactive glycosides were done as described in the previous study (Calis et al. 1999a, 1999b). The air-dried and powdered aerial parts of the plant were extracted twice with $\mathrm{MeOH}-\mathrm{H}_{2} \mathrm{O}(4: 1)$. The water-soluble part of the extract was fractionated by VLC using LiChroprep C-18 as stationary phase and was eluted with increasing amount of $\mathrm{MeOH}$ in $\mathrm{MeOH}-\mathrm{H}_{2} \mathrm{O}$ mixtures. Fractions, which were more than $95 \%$ pure, were monitored by thin layer chromatography (TLC) and similar fractions were combined to yield ten fractions (Calis et al. 1999a). Fractions, which were positive to Kedde reaction, yielded cardio-active glycosides Lanatoside A, Lanatoside C, Glucogitoroside by the help of a series of column chromatography. Fraction eluted with water in VLC was rich in phenylethanoid glycosides. It was further subjected to MPLCon reversed phase material (LiChroprep C-18) and column chromatography as described in previous study to give Lugrandoside, Ferruginosides A and B (Calis et al. 1999b)

\section{Cell lines}

Epithelial-like (Huh7, HepG2) and mesenchymal-like (FOCUS, Mahlavu) human liver cancer cell lines (Buontempo et al. 2011; Yuzugullu et al. 2009) were grown in DMEM with 10\% fetal bovine serum, $1 \%$ non-essential amino acids and $1 \%$ penicillin/streptomycin (GIBCO, Invitrogen) at $37{ }^{\circ} \mathrm{C}$ under $5 \% \mathrm{CO}_{2}$. Cell lines were authenticated with short tandem repeat DNA profiling.

\section{NCI-60 Sulphorhodamine B (SRB) assay}

Cells were grown in 96-well plates (1000-2000 cells/well) for $24 \mathrm{~h}$. Then they were treated with various concentrations of glycosides. After $72 \mathrm{~h}$, cells were fixed using cold, $10 \%$ (v/v) trichloroacetic acid (Merck). $0.4 \%$ (m/v) SRB (Sigma Aldrich) in 1\% acetic acid solution was applied to the plates for $10 \mathrm{~min}$. The unbound SRB was washed with $1 \%$ acetic acid. SRB was then solubilized in $10 \mathrm{mM}$ Tris-base solution. The absorbance was read at $515 \mathrm{~nm}$. The experiments were done in triplicates and $\mathrm{IC}_{50}$ values calculated $\left(R^{2} \geq 0.8\right)$.

\section{Real-time cell growth surveillance by cell electronic sensing}

Human cancer cells (1000-2000 cells/well) were plated onto Eplates and the proliferation curve of the cells was obtained by realtime cell electronic sensing (RT-CES, ACEA). In this experiment, the cell index $(\mathrm{CI})$ values represented cell impedance. Thus, the inhibitory effects of the glycosides in response to DMSO controls were determined by calculating $\mathrm{Cl}_{\text {glycoside }} / \mathrm{Cl}_{\mathrm{DMSO}}$. The experiment was done in triplicate.

\section{Immunofluorescence staining}

Cancer cells were inoculated on coverslips in six-well plates (30,000-100,000 cells/well) and after $24 \mathrm{~h}$, they were treated with the glycosides with $2 \mu \mathrm{M}$ or DMSO controls. Then the cells were fixed with $100 \%$ ice-cold methanol and stained with $1 \mu \mathrm{g} / \mathrm{ml}$ Hoechst (33258, Sigma Aldrich). Finally, the cells were observed under a fluorescent microscope.

\section{Western blot}

Mahlavu cells were treated with either glycosides $(2 \mu \mathrm{M})$ or DMSO control. 20-50 ng of protein was used per well (NuPAGE) for electrophoresis, which were then transferred to nitrocellulose membranes and blotted with specific antibodies (Cleaved caspase8 (9746S Cell Signaling), p-Akt (Ser473) (9271 Cell Signaling), Akt (9272 Cell Signaling), p-ERK1/2(Thr202/Tyr204) (sc16982 Santa 
Cruz), ERK1/2 (sc135900 Santa Cruz), SAPK/JNK (9252 Cell Signaling), pSAPK/JNK (Thr183/Tyr185) (4671 Cell Signaling), p-GSK3$\alpha / \beta$ (Ser21/9) (9331 Cell Signaling), GSK3- $\alpha / \beta$ (sc7291 Santa Cruz), $\mathrm{Na}^{+} / \mathrm{K}^{+}$-ATPase $\alpha 1$ subunit (3010 Cell Signaling), PARP (9532 Cell Signaling) and cleaved caspase-3 (9662S Cell Signaling). Antibodies were diluted in 1:100-500 5\% BSA-TBS-T. Actin (Sigma, A5441), Calnexin (C4731 Sigma Aldrich) antibodies used for equal loading.

\section{Flow cytometry analysis}

Liver cancer cells were treated with the glycosides $(2 \mu \mathrm{M})$ or DMSO control for $24 \mathrm{~h}$ and were then resuspended in Propidium iodide solution for $40 \mathrm{~min}$ (50 $\mu \mathrm{g} / \mathrm{ml}$ PI (Sigma Aldrich), $0.1 \mathrm{mg} / \mathrm{ml}$ RNaseA (Fermentas), 0.05\% Tritron-X-100, 1X PBS). Samples were analyzed using CellQuest software (Becton Dickinson).

\section{Oxidative stress assay}

Cells were inoculated into $100 \mathrm{~mm}$ culture dish (100,000-300,000 cells/dish) for $24 \mathrm{~h}$. Then $2 \mu \mathrm{M}$ Lanatoside C (L2261, Sigma Aldrich) was applied to the cells. One group of cells did not receive the compound but they were grown in selenium deficient serum-free medium as positive control for oxidative stress (Irmak et al. 2003). Third group was treated with DMSO as negative control. After $6 \mathrm{~h}$ and $12 \mathrm{~h}$, the cells were collected and analyzed by MUSE Oxidative Stress Kit, which uses dihydroethidium to monitor superoxide production in the cell (MCH100111, Millipore) (Bindokas et al. 1996). ANNOVA analysis was performed for statistical significance. In parallel, Huh7 and Mahlavu cells were also cultured into 6-well dishes for $24 \mathrm{~h}$ followed by treatment with $2 \mu \mathrm{M}$ Lanatoside $\mathrm{C}$ or DMSO control or selenium deficient serum-free medium. At the end of $6 \mathrm{~h}$ and $12 \mathrm{~h}$ incubation periods, cells were first washed three times with 1X PBS and then dichloro-dihydro-fluorescein diacetate (DCFH-DA) solution in order to detect ROS (particularly $\mathrm{H}_{2} \mathrm{O}_{2}$ ) in the cells ( $10 \mathrm{mM}$ glucose, $1 \mathrm{mM}$ DCFH-DA, 10 mM HEPES dissolved in 1X PBS) (LeBel et al. 1992) was applied onto the cells and they were incubated 15 min in dark, at $37^{\circ} \mathrm{C}$. ROS were observed in situ under the fluorescent microscope.

\section{In vivo animal experiments}

Mahlavu cells $(10,000,000$ cells/mouse) were injected subcutaneously to the flank of nude mice (8-16 weeks old) in DMEM. When tumor volume reached $\sim 150 \mathrm{~mm}^{3}$, drug treatment was initiated. Control group mice (5 mice) received only simple syrup and other subjects received $6 \mathrm{mg} / \mathrm{kg}$ Lanatoside C ( 8 mice) in simple syrup by gavage feeding ( 5 days/week). After 21 days of chemotherapy, nude mice were taken to MRI (acquired with 3-T Siemens MAGNETOM Trio, UMRAM Center, Bilkent University, Ankara Turkey). For statistical significance, Student's $t$-test analysis was performed. Animal care was in agreement with institutional guidelines. Ethical approval was obtained from Bilkent University Animal Ethics Committee (Bilkent University Animal Ethics Committee permit no: 2013/57).

\section{Results}

\section{Cytotoxicities of glycosides purified from Digitalis ferruginea}

Cytotoxic activities of purified glycosides obtained from the $D$. ferruginea (Fig. 1) were initially investigated on both epithelial-like (Huh7, HepG2) and mesenchymal-like (FOCUS, Mahlavu) liver cancer cell lines. The cardiac glycosides Lanatoside A, Lanatoside $C$ and Glucogitoroside showed significant levels of cytotoxicity whereas the phenylpropanoid glycosides had no notable effects on cell growth (Fig. 2a).

Time-dependent cytotoxic activities of cardiac glycosides were examined with the use of RT-CES assay on PTEN adequate Huh7

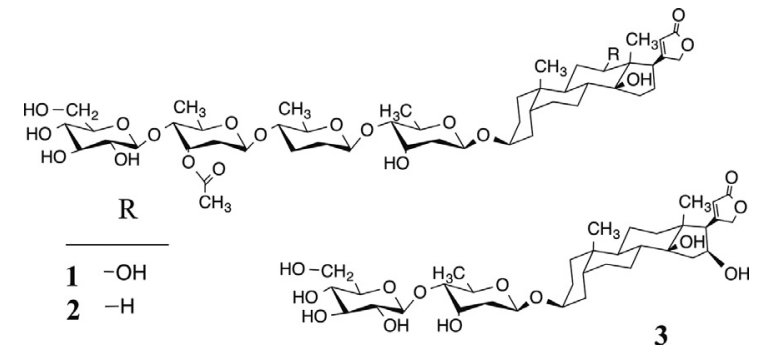

Fig. 1. Chemical structures of the cardiac glycosides, Lanatoside A (1), Lanatoside C (2) and Glucogitoroside (3), which were extracted from Digitalis ferruginea.

cells with normal Akt and ERK proteins and PTEN deficient Mahlavu cells with hyperactive Akt and ERK proteins (Fig. 2b). This assay further confirmed that all three compounds displayed time- and dosedependent growth inhibitory effects (Fig. 2c). Cytotoxic effects of the compounds could be observed after eight hours of treatment, and reached to its highest values upon $72 \mathrm{~h}$ (Fig. 2c). Data obtained from the RT-CES assay on the cells showed a continuous increase on the time course of the cell growth inhibition and hence we evaluated that these compounds should be considered as being cytotoxic rather than cytostatic. Therefore the molecular mechanisms underlying the cytotoxic activities of cardiac glycosides were further analyzed in detail with epithelial like Huh7 cells and PTEN deficient mesenchymal like Mahlavu cells (Fig. 2b).

\section{Characterization of the cell death induced by cardiac glycosides}

Fluorescent-activated cell sorting analysis was performed to analyze cell cycle in the presence of cardiac glycosides. An increase in the $\mathrm{G} 2 / \mathrm{M}$ phase was observed in cells treated with Lanatoside A, Lanatoside $C$ and Glucogitoroside compared to cells in the DMSO controls (Fig. 3a). To explore the death mechanism induced by Lanatoside A, Lanatoside C and Glucogitoroside, Huh7 and Mahlavu cells were treated with these compounds for $48 \mathrm{~h}$. All three compounds caused apoptotic morphological changes in both cell lines by live microscopy and nuclear staining with Hoechst compared to DMSO controls (Fig. 3b). The induction of apoptotic cell death pathway was further confirmed at protein level by western blot analysis of PARP fragmentation. When the Mahlavu cells treated with these compounds were carefully examined, cleavage of PARP and caspase-3 was observed and in the DMSO control neither PARP nor carpase-3cleavage could be observed (Fig. 3c). In conclusion, all these experiments showed that cardiac glycosides Lanatoside A, Lanatoside $\mathrm{C}$ and Glucogitoroside induce caspase-related apoptosis and cell cycle arrest at the G2/M-phase in liver cancer cell lines. Compared to Lanatoside $A$ and Glucogitoroside, Lanatoside $C$ is the drug that is preferably used in clinics for cardiac patients. Thus, we selected and continued detailed molecular analysis of cardiac glycoside induced cell death in liver cancer cells using Lanatoside $\mathrm{C}$.

\section{Oxidative stress induced by Lanatoside $C$}

Cardiac glycosides were previously shown to induce oxidative stress by ROS production by the inhibition of $\mathrm{Na}^{+} / \mathrm{K}^{+}$-ATPase (Prassas and Diamandis 2008). Thus in this study, oxidative stress induction by Lanatoside $C$ was monitored in HCC cells. We showed that Lanatoside C treatment lead to high percentage of ROS (+) cells when compared to DMSO controls in both Huh7 and Mahlavu cells (Fig. 4a). Selenium deficient serum free medium grown cells were used as experimental positive control for ROS generation (Irmak et al. 2003). Treatment with Lanatoside C resulted in about 40\% ROS positivity in both Huh7 and Mahlavu cells (Fig. 4a). In order to visualize in situ presence of oxidative stress, DCFH-DA assay was performed on these cells, which were treated with the Lanatoside $C$ for either $6 \mathrm{~h}$ or $12 \mathrm{~h}$ based on 

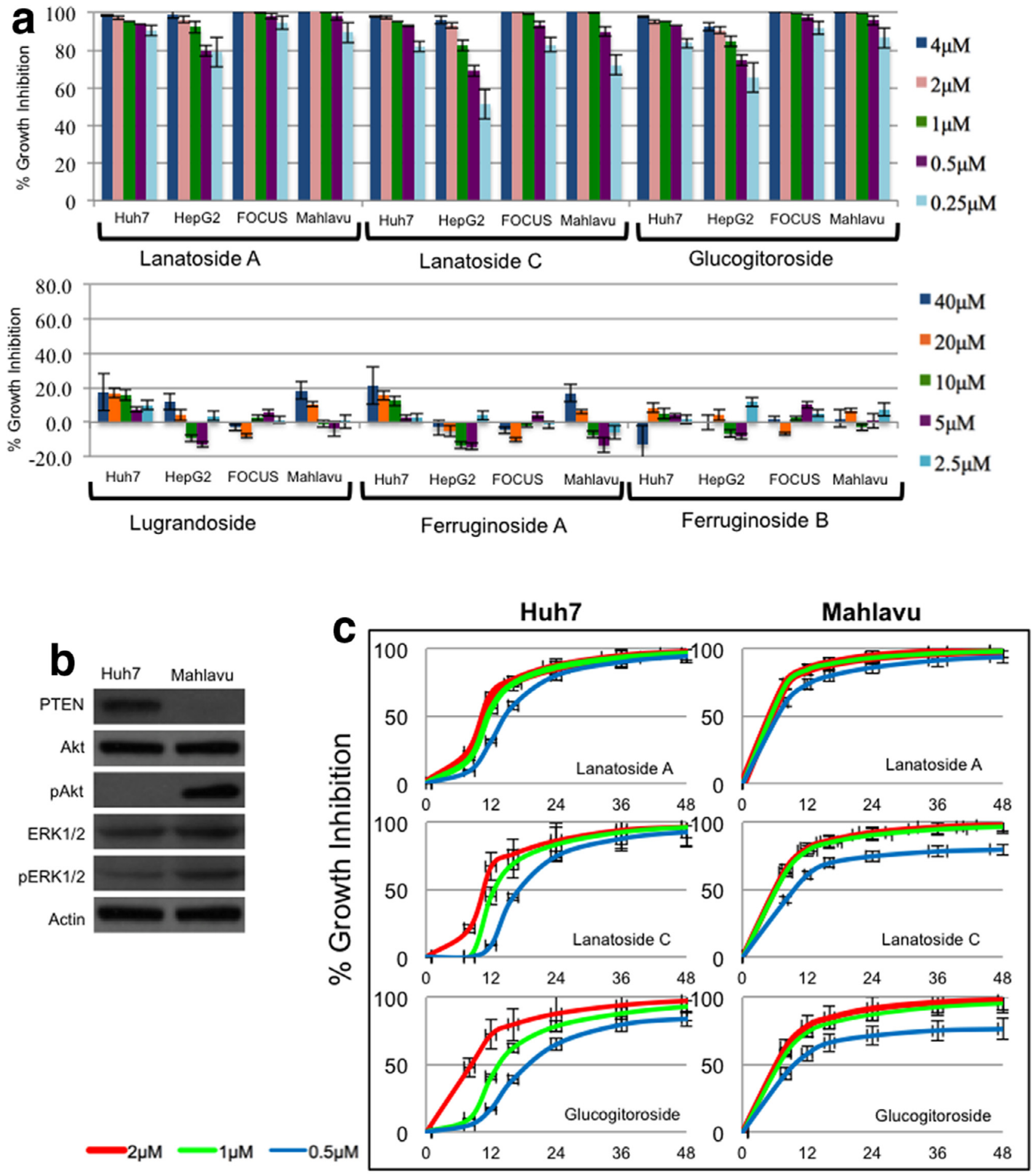

Time (hours)

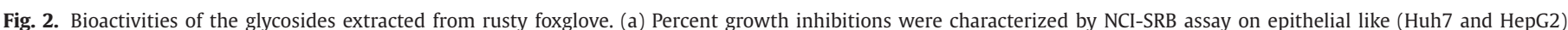

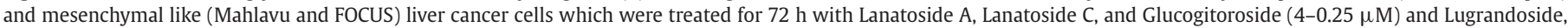

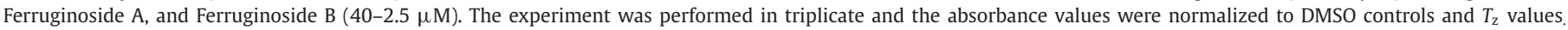

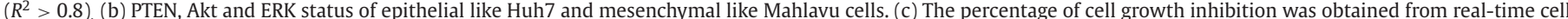

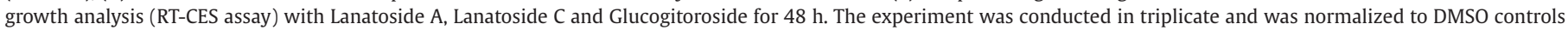
and $T_{\mathrm{Z}}\left(R^{2}>0.8\right)$. 


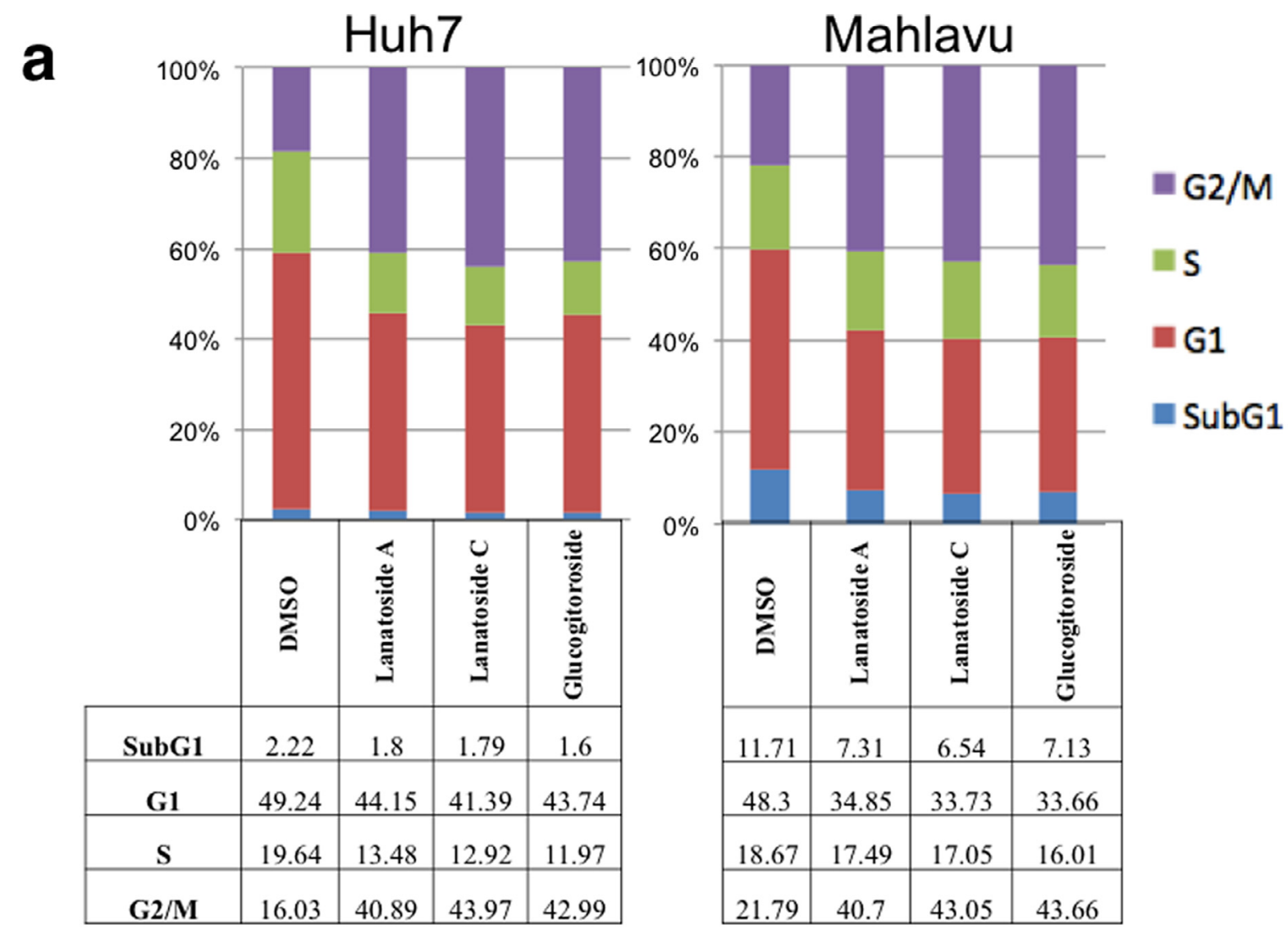

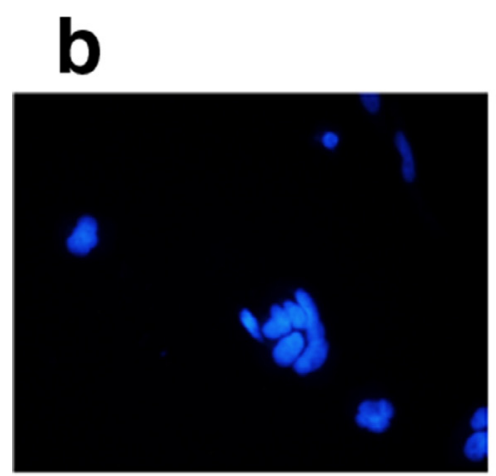

\section{Lanatoside A}

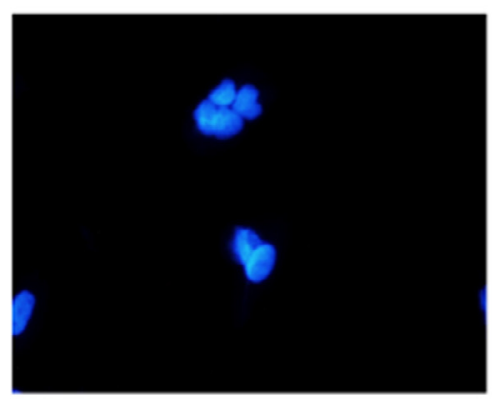

\section{Glucogitoroside}

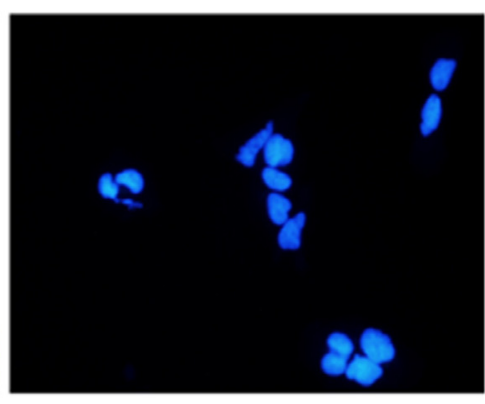

Lanatoside C

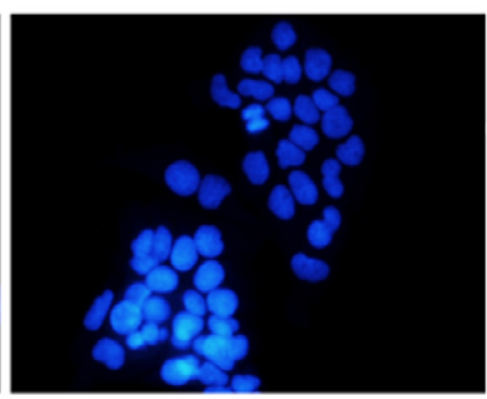

Cleavedcaspase-3

\section{Actin}

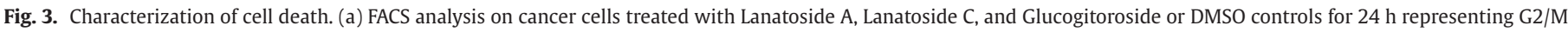

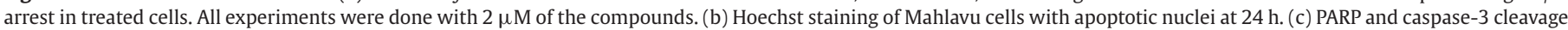
in Mahlavu cells treated with the glycosides for $48 \mathrm{~h}$. Actin was used for equal loading. 

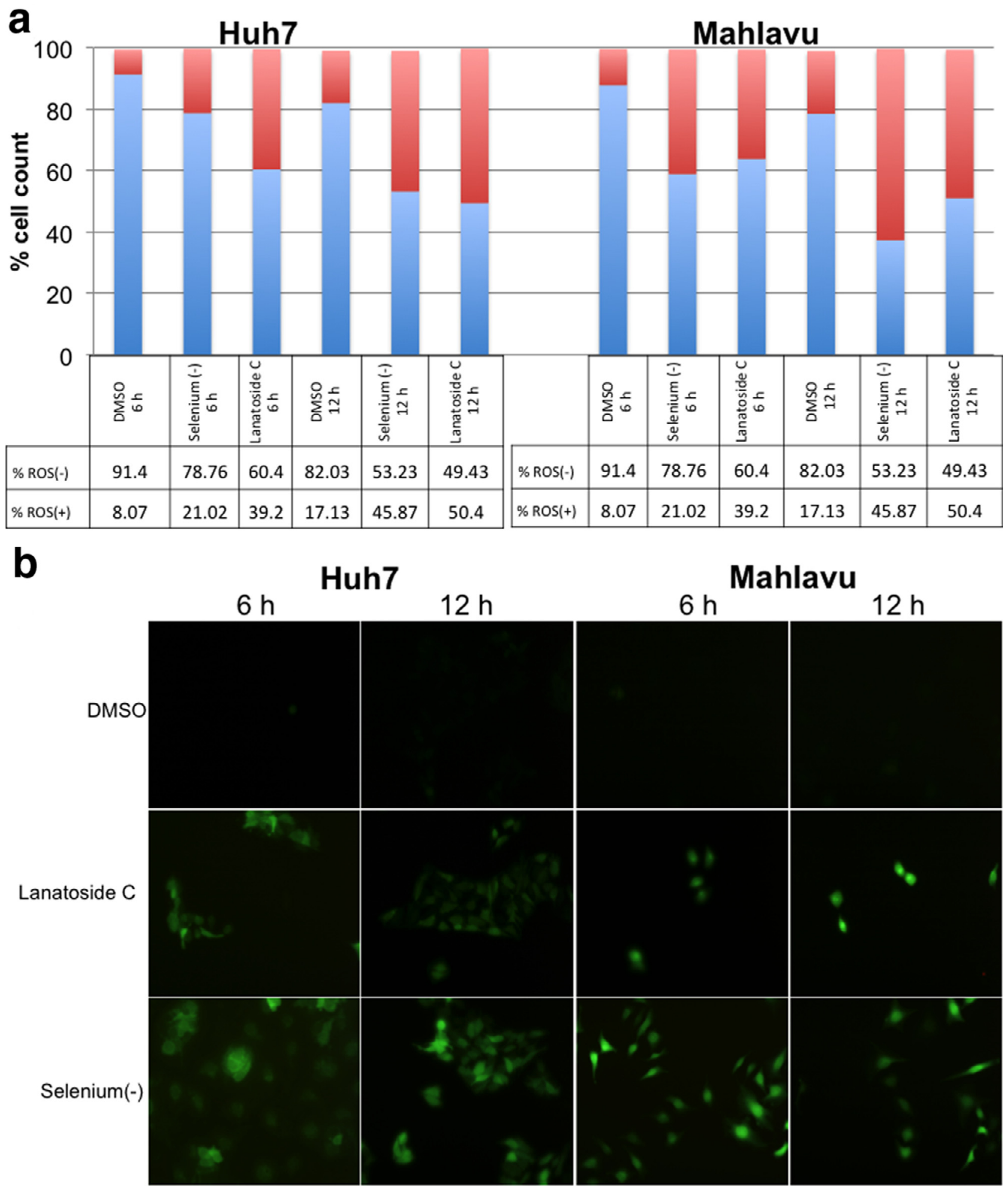

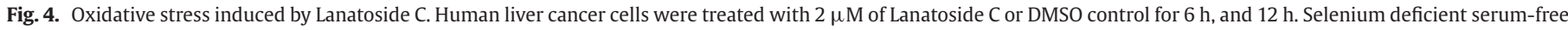

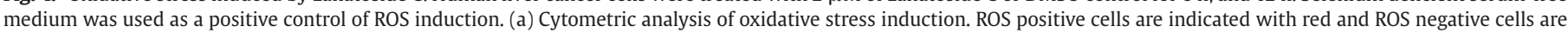

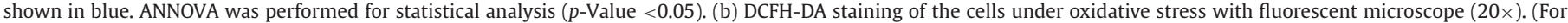
interpretation of the references to color in this figure legend, the reader is referred to the web version of this article.)

RT-CES experiment results that indicated the start of cell death as early as $8 \mathrm{~h}$ (Fig. 2). In the presence of oxidative stress DCFH-DA dye was oxidized to a green fluorescent molecule, DCF. Fluorescent microscopy images showed that oxidative stress triggered by Lanatoside C as early as 6-12 hours in parallel to cell death (Fig. 4b).
Cellular pathways targeted by Lanatoside $C$

Based on the finding that Lanatoside $C$ caused ROS accumulation, targets of ROS at the protein level was further investigated by western blot analyses. ERK, Akt and JNK proteins were previously shown to 

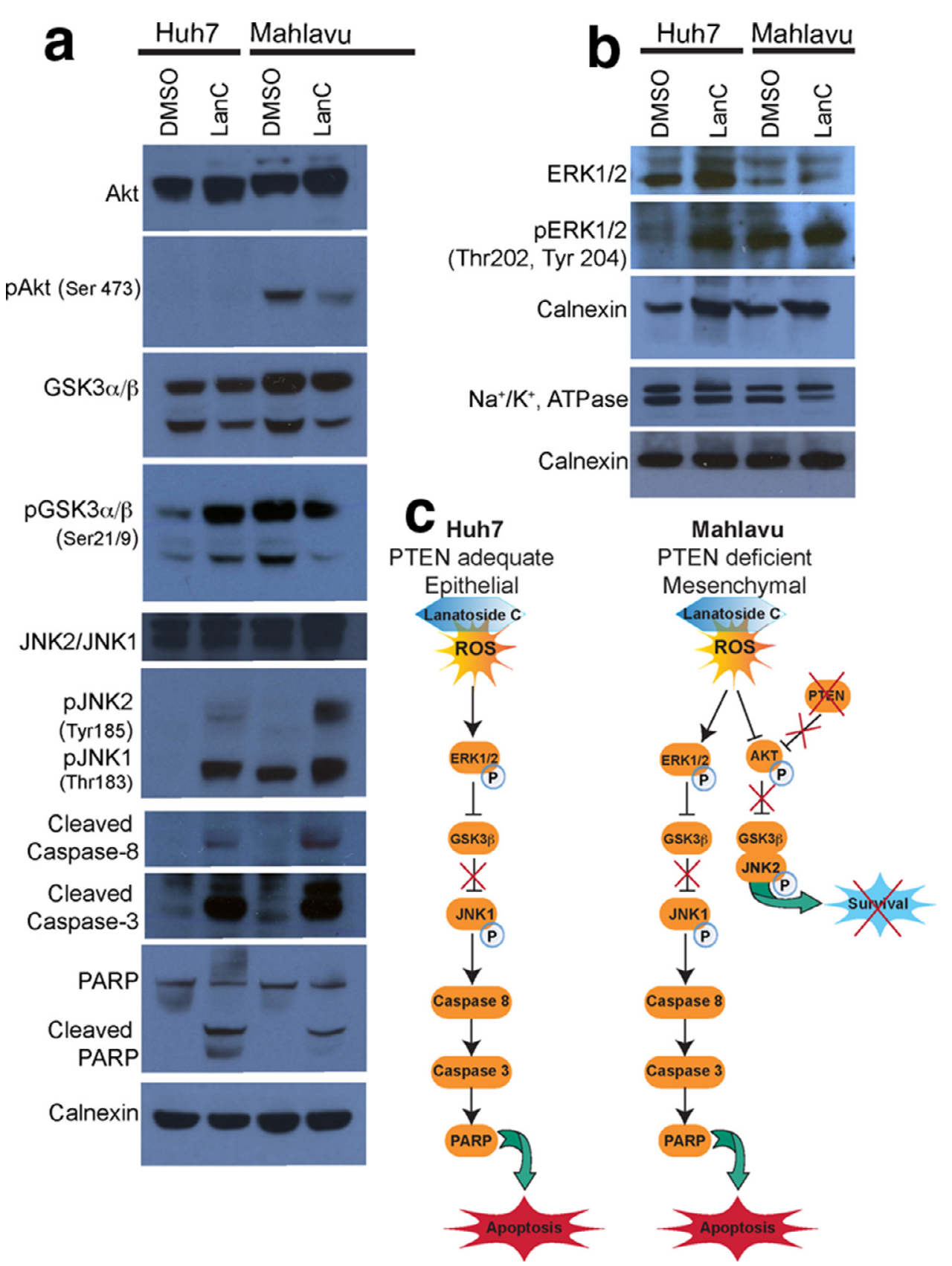

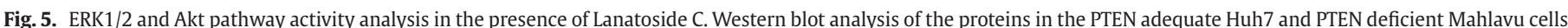

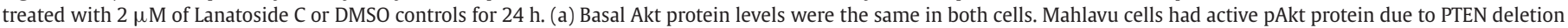

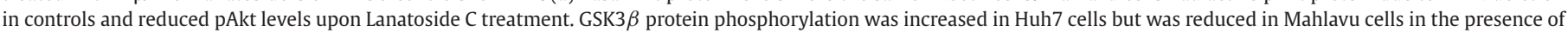

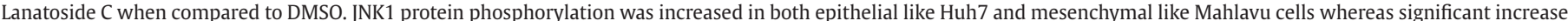

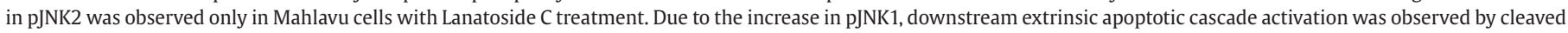

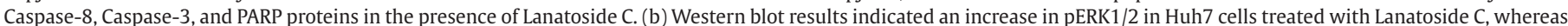

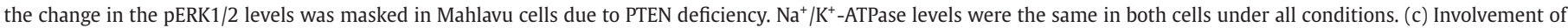
differentially altered ERK1/2 and Akt pathways in response to Lanatoside C treatment in PTEN adequate and deficient liver cancer cells.

be involved in the oxidative stress induced cellular pathways (Cagnol and Chambard 2010). Therefore these pathways were initially investigated in the presence of Lanatoside C (Fig. 5 and Suppl. Figs. 1-4). Lanatoside $\mathrm{C}$ increased the phosphorylation of JNK1 induced caspase8 dependent apoptotic cascade in both Huh7 and PTEN deficient Mahlavu cells (Fig. 5a and Suppl. Fig. 1). Because JNK induced apoptosis was dependent on GSK3 $\beta$ in pancreas cancer cells, we then analyzed the upstream elements of this pathway, which includes GSK3 $\beta$, Akt and ERK. In PTEN adequate epithelial like Huh7 cells, ROS accumulation due to Lanatoside $C$ treatment lead to an increase in both ERK and GSK3 $\beta$ phosphorylation. However, in PTEN deficient mes- enchymal like Mahlavu cells, ROS initiated the dephosphorylation of Akt, leading to a decrease in GSK3 $\beta$ phosphorylation and an increase in both JNK2 and JNK1 phosphorylation (Fig. 5a and b). On the other hand, there was no significant JNK2 phosphorylation in Huh7 cells, which displayed normal PI3K/Akt activities. Furthermore, we verified the presence of previously described target of cardiac glycosides, $\mathrm{Na}^{+} / \mathrm{K}^{+}$-ATPase and both Huh7 and Mahlavu cells expressed this protein (Fig. 5b). The mechanism of action of Lanatoside $C$ in PTEN adequate Huh7 and PTEN deficient/Akt hyperactive Mahlavu cells are discussed in detail with respect to genetic backgrounds of these cells below (Fig. 5c). 

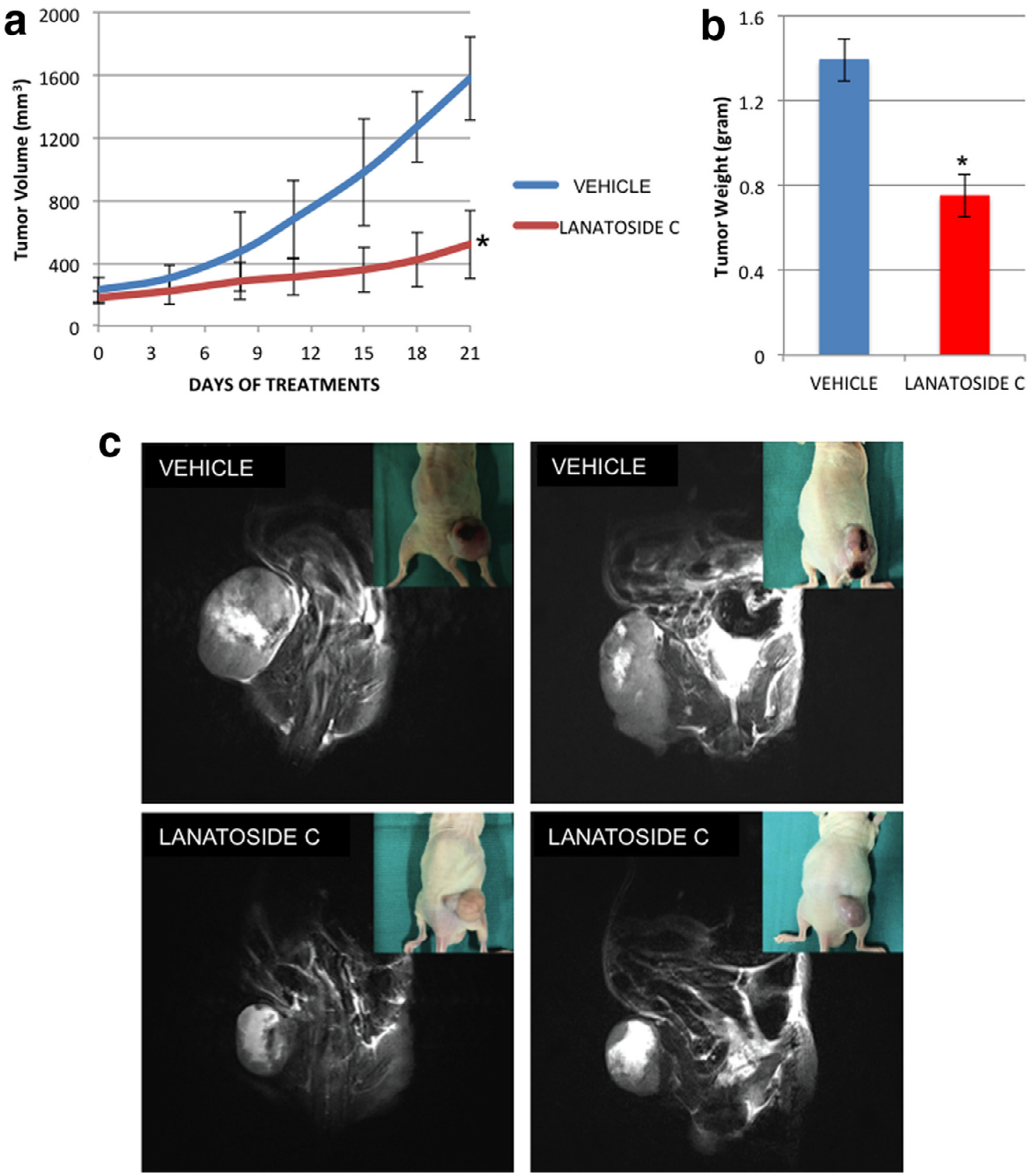

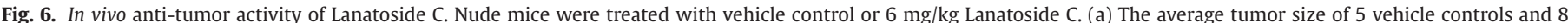

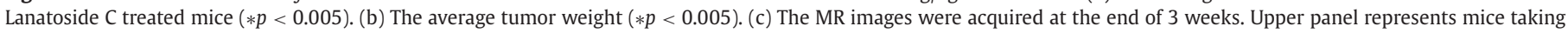
vehicle and lower is for mice taking Lanatoside C. Tumor sizes were tracked twice a week. Student's $t$-test was performed for statistical analysis.

In vivo anti-tumor effects of Lanatoside C on Mahlavu mice xenograft models

Finally, the anti-tumor activity of Lanatoside $\mathrm{C}$ was investigated on nude mice tumor xenografts. Lanatoside $C$ exhibited chemotherapeutic behaviors. Mice taking Lanatoside C ( 8 mice) had significant reduction in tumor volume at the end of 21 days whereas the average tumor volume of control group ( 5 mice) had reached $1600 \mathrm{~mm}^{3}$ (Fig. 6a). Tumor weight was also reduced by almost $50 \%$ in mice taking Lanatoside C compared to vehicle controls (Fig. 6b). Furthermore, the heterogeneity of the images from MRI analysis indicated the xenograft tumor tissue bared extensive necrosis in Lanatoside $\mathrm{C}$ treated mice compared to vehicle controls (Fig. 6c).

\section{Discussion}

Cardiac glycosides are among one of the oldest medicines and have been in use for centuries to treat heart failure (Prassas and Diamandis 2008). They are steroid-like compounds, which inhibit $\mathrm{Na}^{+} / \mathrm{K}^{+}$-ATPase and indirectly regulate cell signaling. This study investigated the molecular mechanisms of the cytotoxic effects of cardiac glycosides obtained from $D$. ferruginea on liver cancer cells in which the treatment required novel chemotherapeutic agents. Due to various etiological factors, liver cancer has a heterogeneous genetic background, which leads to the alterations of cellular signals. For this purpose, initially, growth inhibitory effects of glycosides were investigated on epithelial like (Huh7 and HepG2) and mesenchymal like (Mahlavu and FOCUS) liver cancer cells (Figs. 2 and 3). Three of the 
six extracts displayed high cytotoxicity to liver cancer cells at nanomolar concentrations, which were reported not to be cardio-toxic (Newman et al. 2008). While the proteins under the influence of cardiac glycosides were reported in various cancers with the exception of liver cancer, the comprehensive signaling analysis was not studied in the context of epithelial vs. mesenchymal background (Newman et al. 2008). In this study, drug resistant PTEN deficient mesenchymal like Mahlavu liver cancer cells and drug sensitive PTEN adequate Huh7 cells were selected to be studied in depth including the molecular pathways involved in cell death in the presence of cardiac glycoside Lanatoside C (Buontempo et al. 2011; Wei et al. 2013; Yuzugullu et al. 2009).

Cardiac glycosides have been reported to activate apoptosis or autophagy-induced cell death in a tissue-specific manner (Wang et al. 2014; Xie and Cai 2003). In this study, we characterized liver cancer cell death as apoptosis. Moreover, these compounds induced G2/M cell cycle arrest in both Huh7 and Mahlavu liver cancer cells (Fig. 3). It is known that when glycosides translocate into cytoplasm, $\mathrm{Na}^{+} / \mathrm{K}^{+}$ATPase pumps become inactive. In parallel, ROS accumulation takes place. Upon ROS accumulation, ERK1/2 and JNK proteins are activated (Prassas and Diamandis 2008). Lanatoside C was selected to further investigate the molecular mechanisms, because this compound has been preferably used in clinics. Lanatoside $C$ also promoted the accumulation of ROS in liver cancer cells, as reported with other glycosides such as Bufalin and Ouabain in other cancer cells (Fig. 4). We then identified the pathways targeted by Lanatoside $C$ in the context of epithelial vs. mesenchymal background. Our data with Lanatoside $\mathrm{C}$ on epithelial like Huh7 are also in correlation with the previous findings. Activation of ERK1/2 phosphorylates GSK3 $\beta$, which lead to its inactivation, hence the activation of JNK protein occurs (Marchand et al. 2012). Huh7 cells under Lanatoside $C$ treatment also displayed active ERK1/2 and inactive GSK3 $\beta$ and as a result activation of JNK1 observed, in parallel caspase-8 cascade induction occurred (Fig. 4). Cell death by apoptosis can be classified as being intrinsic or extrinsic. Their downstream differential caspase activation is an indicator of the apoptotic pathway induction type (Fulda and Debatin 2006). Caspase-8 cleavage is associated with the extrinsic pathway. Here, we showed the cleavage of caspase- 8 and its downstream targets caspase-3 and PARP proteins under Lanatoside $\mathrm{C}$ treatment (Fig. 5a and $\mathrm{c})$.

$\operatorname{GSK} 3 \beta$, which acts as a molecular effector in this pathway, was previously shown to both trigger and inhibit apoptotic pathways (Doble and Woodgett 2003). Un-phosphorylated form of GSK3 $\beta$ is involved in the activation of caspase- 9 dependent intrinsic pathway and death-receptor initiated extrinsic apoptosis pathway is inhibited by this protein (Fulda and Debatin 2006). Thus our data illustrated that phosphorylation of GSK3 $\beta$ and hence inactivation of this protein together with the presence of cleaved caspase- 8 and -3 was due to Lanatoside C's induction of extrinsic apoptotic pathway in epithelial like liver cancer cell Huh7 through JNK1 activation (Fig. 5). However, PTEN deficient mesenchymal like Mahlavu cells did not exhibit a significant change in the phosphorylation of ERK1/2 when compared to DMSO controls. Furthermore, these cells have already activated ERK $1 / 2$ and pGSK3 $\beta$ levels. In this and in our previous studies, we showed that PI3K/Akt pathway was constitutively hyperactive in Mahlavu cell line due to PTEN deficiency (Fig. 2) (Buontempo et al. 2011). Therefore the ROS-induced ERK1/2 phosphorylation was masked by the hyperactivity of the Akt pathway (Fig. 5). Reduction in inactive $\operatorname{pGSK} 3 \beta$ due to the decrease in pAkt in Mahlavu cells treated with Lanatoside $C$ is an opposing factor for cell survival (Fig. 5). Active GSK3 $\beta$ and JNK2 were previously shown to be involved in prevention of cell survival (Hu et al. 2009). Similarly increase in the levels of phospho-JNK2 and decrease in the levels of phospho-GSK3 $\beta$ were observed in Mahlavu cells treated with Lanatoside C, in favor of the prevention of cell survival (Fig. 5). Our comparative data with drug resistant PTEN deficient mesenchymal like Mahlavu and drug sen- sitive PTEN adequate epithelial like Huh7 liver cancer cells demonstrated that cardiac glycoside Lanatoside $C$ can also induce cell death by two parallel pathways. One is the induction of ERK1/2 dependent apoptosis and the other is inhibition of Akt dependent cell survival by differential activation of JNK1 and JNK2 respectively. Furthermore the results of the in vitro study are also correlated with in vivo data signifying the potential of Lanatoside $C$ as liver cancer therapeutics independent of the PTEN tumor suppressor protein status. The lack of PTEN protein is highly correlated with the aggressive behavior of this cancer due to Akt pathway activation and cell survival. The present study proposes that clinically preferred glycoside Lanatoside $C$ can be repurposed as a chemotherapeutic supplementary agent towards the liver cancer with both normal- and hyper-active Akt pathway.

\section{Conflict of interest}

The authors declare no conflicts of interest.

\section{Acknowledgments}

The authors thank Dr. Ali Osmay Gure for discussions about the final version of the manuscript, Bilge Ozturk and Gamze Aykut, DVM for laboratory assistance and Ms. R. Nelson, M. Atalay (U. Chicago) and A.S. Kurt (U. King's College London) for edition of the manuscript in English.

\section{Supplementary materials}

Supplementary material associated with this article can be found, in the online version, at doi:10.1016/j.phymed.2015.11.012.

\section{References}

Baffy, G., Brunt, E.M., Caldwell, S.H., 2012. Hepatocellular carcinoma in non-alcoholic fatty liver disease: an emerging menace. J. Hepatol. 56, 1384-1391.

Bindokas, V.P., Jordan, J., Lee, C.C., Miller, R.J., 1996. Superoxide production in rat hippocampal neurons: selective imaging with hydroethidine. J. Neurosci. 16, 13241336.

Buontempo, F., et al., 2011. Inhibition of Akt signaling in hepatoma cells induces apoptotic cell death independent of Akt activation status. Investig. New Drugs 29, 13031313.

Cagnol, S., Chambard, J.-C., 2010. ERK and cell death: mechanisms of ERK-induced cell death - apoptosis, autophagy and senescence. FEBS J. 277, 2-21.

Calis, I, et al., 1999a. Phenylethanoid and cardioactive glycosides from Digitalis ferruginea. Pharmazie 54 (12), 926-930.

Calis, I, et al., 1999b. Phenylethanoid glycosides from Digitalis ferruginea subsp. ferruginea. Chem. Pharm. Bull. 47 (9), 1305-1307.

Doble, B.W., Woodgett, J.R., 2003. GSK-3: tricks of the trade for a multi-tasking kinase. J. Cell Sci. 116, 1175-1186.

El-Serag, H.B., et al., 2008. Diagnosis and treatment of hepatocellular carcinoma. Gastroenterology 134, 1752-1763.

Ersahin, T., Tuncbag, N., Cetin-Atalay, R., 2015. The PI3K/AKT/mTOR interactive pathway. Mol. Biosyst. 11 (7), 1946-1954.

Ferlay J., Soerjomataram I., Ervik M., et al., 2012. Cancer Incidance and Mortality Worldwide: IARC Cancer Base No. 11 [Internet]. Lyon: IARC. GLOBOCAN 1.0.

Fulda, S., Debatin, K.-M., 2006. Extrinsic versus intrinsic apoptosis pathways in anticancer chemotherapy. Oncogene 25, 4798-4811.

$\mathrm{Hu}$, D., et al., 2009. GSK3beta is involved in JNK2-mediated beta-catenin inhibition. PLoS One 4, e6640.

Irmak, M.B., et al., 2003. Acquired tolerance of hepatocellular carcinoma cells to selenium deficiency: a selective survival mechanism? Cancer Res. 63, 6707-6715.

Ke, A.-W., et al., 2011. CD151 amplifies signaling by integrin $\alpha 6 \beta 1$ to PI3K and induces the epithelial-mesenchymal transition in HCC cells. Gastroenterology 140 pp. 1629-41.e15.

LeBel, C.P., Ishiropoulos, H., Bondy, S.C., 1992. Evaluation of the probe $2^{\prime}, 7^{\prime}$ dichlorofluorescin as an indicator of reactive oxygen species formation and oxidative stress. Chem. Res. Toxicol. 5, 227-231.

Liu, L., et al., 2007. Association of PI3K-Akt signaling pathway with digitalis-induced hypertrophy of cardiac myocytes. Am. J. Physiol.: Cell Physiol. 293, C1489-C1497.

Marchand, Tremblay, I., Cagnol, S., B.M., 2012. Inhibition of glycogen synthase kinase3 activity triggers an apoptotic response in pancreatic cancer cells through JNKdependent mechanisms. Carcinogenesis 33 (3), 529-537.

Newman, R.A., et al., 2008. Cardiac glycosides as novel cancer therapeutic agents. Mol. Interv. 8, 36-49.

Prassas, I., Diamandis, E.P., 2008. Novel therapeutic applications of cardiac glycosides. Nat. Rev. Drug Discov. 7, 926-935. 
Shearn, C.T., Smathers, R.L., Stewart, B.J., Fritz, K.S., Galligan, J.J., Hail, N., Petersen, D.R., 2011. Phosphatase and tensin homolog deleted on chromosome 10 (PTEN) inhibition by 4-hydroxynonenal leads to increased Akt activation in hepatocytes. Mol. Pharmacol. 79, 941-952.

Wang, Y., et al., 2014. Src mediates extracellular signal-regulated kinase 1/2 activation and autophagic cell death induced by cardiac glycosides in human non-small cell lung cancer cell lines. Mol. Carcinog. 54 (S1), E26-34.

Wei, Z., Doria, C., Liu, Y., 2013. Targeted therapies in the treatment of advanced hepatocellular carcinoma. Clin. Med. Insights. Oncol. 7, 87-102.
Xie, C.-M., et al., 2011. Bufalin induces autophagy-mediated cell death in human colon cancer cells through reactive oxygen species generation and JNK activation. Free Radic. Biol. Med. 51, 1365-1375.

Xie, Z., Cai, T., 2003. $\mathrm{Na}^{+}-\mathrm{K}^{+}$-ATPase-mediated signal transduction: from protein interaction to cellular function. Mol. Interv. 3, 157-168.

Yau, T., et al., 2009. Management of advanced hepatocellular carcinoma in the era of targeted therapy. Liver Int. 29, 10-17.

Yuzugullu, H., et al., 2009. Canonical Wnt signaling is antagonized by noncanonical Wnt5a in hepatocellular carcinoma cells. Mol. Cancer 8, 90. 